\title{
Midgut-Specific Expression of P450 Gene Increases Deltamethrin Tolerance in The Fall Armyworm, Spodoptera Frugiperda
}

Subba Reddy Palli ( $\nabla$ rpalli@uky.edu )

University of Kentucky https://orcid.org/0000-0002-0873-3247

\section{Xien Chen}

University of Kentucky

\section{Research Article}

Keywords: Midgut-specific genes, promoters, transgenesis, P450, deltamethrin, and Spodoptera frugiperda

Posted Date: November 8th, 2021

DOI: https://doi.org/10.21203/rs.3.rs-996825/v1

License: (c) (i) This work is licensed under a Creative Commons Attribution 4.0 International License. Read Full License 


\section{Abstract}

The piggyBac-based germline transformation system was recently established in a global agricultural pest, the fall armyworm (FAW), Spodoptera frugiperda. Tissue-specific promoters are needed to apply this transformation system to express transgenes in a tissue-specific manner. Highly expressed genes in the midgut were identified by RNA sequencing and RT-qPCR. Promoter regions of 11 genes highly expressed in the midgut were identified and cloned. Baculoviruses expressing the luciferase under the control of these promoters were produced and tested in the FAW. These baculoviruses did not show significant luciferase activity in the FAW midgut. Four transgenic FAW lines, expressing the luciferase under the control of SfSP38/P2000, SfCalphotin/P2000, SfMG17/P2000, and SfCPH38/P2000 promoters were generated using piggyBac-based germline transformation methods. Significantly higher luciferase activity was detected in the midgut than in other tissues of transgenic FAW. SfCPH38/P2000 promoter with the highest activity and midgut-specificity was used to drive the expression of a P450, SfCYP321A8 known to be involved in deltamethrin resistance. Higher mRNA levels of SfCYP321A8 and P450 activity were detected in the midgut of transgenic larvae than in wild-type larvae. Bioassays showed that the transgenic larvae expressing SfCYP321A8 in the midgut are tolerant to deltamethrin. Here, we presented methods for the identification of midgut-specific promoters in the FAW and used them to study the role of P450 overexpression in the midgut on insecticide resistance. These methods could also be used to identify other tissue-specific promoters for applications of piggyBac-based germline transformation in functional genomics in FAW and other non-model insects.

\section{Highlights}

1. Midgut-specific promoters were identified in a major pest insect

2. Midgut-specific promoters were used to express P450 gene

3. Overexpression in the midgut increased deltamethrin tolerance

4. The methods could be used to identify tissue-specific promoters in other non-model insects.

\section{Introduction}

The midgut is the second largest organ and site of digestion of ingested food and absorption of nutrients (Hakim et al. 2010). As an important target tissue for many insecticides, insect midgut also plays a major role in insecticide resistance (Smagghe and Tirry 2001). It is also an immune organ acting as the first line of defense against pathogens, including bacteria and viruses (Hakim et al. 2010). The distinctive environment in the midgut also allows microbial colonization, which benefits the host insects (Engel and Moran 2013). Additionally, remodeling of the midgut is critical for successful metamorphosis in insects (Parthasarathy and Palli 2007; Wu et al. 2006). It is believed that the products from the midgut genes, especially the highly expressed genes, and midgut-specific genes, perform critical functions (Hakim et al. 2010). Studies on Drosophila melanogaster extended our knowledge on insect midgut (Harrop et al. 2014; Hung et al. 2020; Lucchetta and Ohlstein 2012). However, these results may not apply to all insects with 
significant differences in structure and function of the midgut. The transgene-based overexpression, knockdown, and knockout of midgut genes used routinely in D. melanogaster are still challenging in other insects. Availability of these methods in pest insects will promote studies on the midgut and benefit management of these pests.

The fall armyworm (FAW), Spodoptera frugiperda (Lepidoptera: Noctuidae), is native to tropical and subtropical regions of the Americas. It is now successfully invaded most parts of the world (Gui et al. 2020). As a successful polyphagous pest, FAW feeds on more than 300 species of host plants, including some major food crops (Montezano et al. 2018). The FAW has developed resistance to many chemical insecticides (Carvalho et al. 2013; Diez-Rodriguez 2001), and transgenic Bt corn (Omoto et al. 2016). The midgut is likely to play a central role in FAW feeding on multiple plants and developing insecticide resistance. However, there are not many studies on FAW midgut.

Unlike in model insects, the germline transformation systems are not well developed in lepidopteran pest insects. We recently established a piggyBac-based transformation system in the FAW (Chen et al. 2020b). Several highly active promoters have been identified in FAW (Bleckmann et al. 2015; Chen et al. 2020a), which will further facilitate the use of the piggyBac system in FAW. However, tissue-specific promoters are needed to conduct tissue-specific gain- or loss-of-function studies using the piggyBac-based transformation system. There are no reports on the FAW tissue-specific promoters.

In an attempt to develop tools for tissue-specific gain- or loss-of-function studies in FAW, we analyzed RNA-Seq data from the midgut and other tissues, identified several genes uniquely, and highly expressed in the midgut. Promoter regions of identified genes were used to drive the luciferase reporter gene expression in cells via the baculovirus system. In addition, transgenic reporter FAW lines were established to investigate the candidate promoter activity in vivo. Finally, the promoter of SfCHP38 gene, which is highly active in the midgut compared to that in other tissues, was selected to drive the expression of a P450 gene, SfCYP321A8, involved in deltamethrin resistance. Deltamethrin tolerance was observed in transgenic animals expressing SfCYP321A8 in the midgut.

\section{Materials And Methods}

\section{Insect and cells}

The laboratory strain of FAW was purchased from Benzon Research Inc. (Pennsylvania, USA) and reared for multiple generations without exposure to any pesticides. Larvae were fed on artificial diet from Southland Product Inc. (Arkansas, USA). Adults were provided with a $10 \%$ sucrose solution under the conditions of $23 \pm 1{ }^{\circ} \mathrm{C}, 75 \pm 5 \%$ relative humidity and a photoperiod of $16: 8$ (light: dark). Sf9 cells were cultured at $27^{\circ} \mathrm{C}$ in Sf-900 II medium (Thermo Fisher, USA). SfMG_0617 (hereafter referred to as Sf17) cell line, generated from FAW midgut (Zhou et al. 2020), was maintained in TNM-FH insect medium (Sigma-Aldrich, USA) supplemented with $10 \%$ FBS at $27^{\circ} \mathrm{C}$.

\section{Plasmid construction}


The candidate midgut gene promoters were identified and cloned as described in our previous publication (Chen et al. 2020a). To make the plasmids for producing baculovirues, the ORF of luciferase gene was amplified from the pG5Luc vector using the primers containing $20 \mathrm{bp}$ homologous arms on either end of EcoR I/ Kpn I digested pFastBac-S/His vector and cloned into $\mathrm{pFastBac-S/His} \mathrm{vector} \mathrm{by} \mathrm{Gibson} \mathrm{assembly}$ (NEB, USA). Promoters of FAW midgut-specific genes, containing $20 \mathrm{bp}$ homologous arms on either end of SnaB I/BamH I digested pFastBac-Luc plasmid were amplified from FAW genomic DNA, and inserted into SnaB I/BamH I digested pFastBac-Luc plasmid to generate pFastBac-promoter-Luc vectors as described above. All vectors were transformed into E. coli10G cells (Lucigen, USA). Positive transformants were selected and cultured in $3 \mathrm{ml}$ LB medium. The plasmid DNAs were extracted using the plasmid mini kit (Qiagen, USA)

To prepare the plasmids for generating transgenic FAW, a piggyBac vector, pBac:hr5ie1-EGFPSV40:hr5ie1-Cas9-SV40 (gifted from Dr. Tan, Shanghai institution of plant physiology and ecology, China), were digested with Aat II/Apa I to remove the Cas9 cassette. The ORF of luciferase or a P450 gene, SfCYP321A8, from FAW was inserted into the digested piggyBac vector as described above to produce the intermediate vectors pBac:hr5ie1-EGFP-SV40:hr5ie1-Luc-SV4 and pBac:hr5ie1-EGFPSV40:hr5ie1-SfCYP321A8-SV40. The cloned promoters were then inserted into Kpn I/Aat II or Xho I/Aat II digested intermediate vectors to generate the final vectors, pBac:hr5ie1-EGFP-SV40:hr5/Promoter-LucSV40, pBac:ie1-EGFP-SV40:Promoter-Luc-SV40, pBac:hr5ie1-EGFP-SV40:hr5/Promoter-SfCYP321A8SV40, and pBac:ie1-EGFP-SV40: Promoter-SfCYP321A8-SV40. All constructs were transformed into E. coli $10 \mathrm{G}$ cells. Positive transformants were selected and cultured in $100 \mathrm{ml}$ LB medium. The plasmid DNAs for transfection were prepared using the plasmid midi kit (Qiagen, USA).

Primers used to produce constructs are listed in Table S1.

\section{Reporter baculovirus preparation}

pFastBac-promoter-Luc plasmids were extracted and transformed into E. coli DH10Bac cells (Invitrogen, USA) to produce recombinant bacmids, which were then used to generate reporter baculoviruses in Sf9 cells following the methods described in bac-to-bac system (Invitrogen, USA). The viral nucleic acids of P2 viruses were purified using the PureLink ${ }^{T M}$ Viral RNA/DNA Mini Kit (Invitrogen, USA). The plaqueforming units (PFU) of reporter baculoviruses were determined using BacPAK qPCR Titration Kit (Takara Bio, USA).

\section{RNA isolation, cDNA preparation and RT-qPCR}

Total RNA was isolated using TRI reagent (Molecular Research Center Inc., Cincinnati, $\mathrm{OH}$ ), and converted to cDNA using the M-MLV Reverse Transcriptase (Invitrogen, USA). Real-time PCR reactions were conducted in a 10- $\mu \mathrm{L}$ total reaction volume containing $5 \mu \mathrm{L}$ of $2 x S Y B R$ Mix (BioRad, USA), $0.4 \mu \mathrm{L}$ of each primer, $0.8 \mu \mathrm{L}$ of 20 -fold diluted cDNA, and $3.4 \mu \mathrm{L}$ of double-distilled water. Primers were designed using Primer3 (http://bioinfo.ut.ee/primers-0.4.0/) and listed in Table S1. The reaction conditions used were as follows: $95^{\circ} \mathrm{C}$ for $2 \mathrm{~min}, 40$ cycles of $95^{\circ} \mathrm{C}$ for 10 seconds, and $60^{\circ} \mathrm{C}$ for $1 \mathrm{~min}$. The expression levels of 
each gene were calculated as a fold change over the expression levels of a reference gene, 28S rRNA. All samples contain three biological replicates.

\section{Transformation of FAW}

The hyperactive transposase mRNA was prepared as described previously (Chen et al. 2020b), and stored at $-80^{\circ} \mathrm{C}$. A mixture of hyperactive transposase mRNA $(400 \mathrm{ng} / \mu \mathrm{L})$ and each final vector $(300 \mathrm{ng} / \mu \mathrm{L})$ was injected into fresh FAW embryos (less than $4 \mathrm{hr}$ old) as previously described (Chen et al. 2020b). The survived larvae (G0) were reared under normal conditions. Collection and screening of newly hatched $1^{\text {st }}$ instar larvae (G1) were performed as described previously (Chen et al. 2020b). All transgenic larvae and adults were reared under normal conditions.

\section{Luciferase activity assay}

To test the luciferase activity of reporter baculoviruses in cell lines, Sf9 and Sf17 cells were seeded in 96well culture plates at a density of $2 \times 10^{5}$ cells per well and incubated at $27{ }^{\circ} \mathrm{C}$ overnight. $6 \times 10^{4} \mathrm{PFU}$ of each virus was added to $100 \mu \mathrm{L}$ medium of Sf17 and Sf9 cells. Cells were collected for luciferase activity assay at $24 \mathrm{hr}$ post-infection and the luciferase activity was quantified as described (Chen et al. 2020a). Four biological replicates were performed for each treatment.

To determine the luciferase activity in the midgut and other tissues of FAW larvae, newly molted $6^{\text {th }}$ instar larvae were injected with $2 \times 10^{5} \mathrm{PFU}$ of each virus. Midguts and other tissues dissected on the third-day post-injection were homogenized in the luciferase assay buffer and centrifuged at $12,000 \mathrm{rpm}, 4^{\circ} \mathrm{C} .20 \mu \mathrm{L}$ and $10 \mu \mathrm{L}$ of each supernatant were used for luciferase activity assay and protein concentration determination, respectively, as described previously (Chen et al. 2020a). Three biological replicates were performed for each treatment.

To investigate the luciferase activity in tissues of transgenic reporter FAW larvae, four different tissues, including head, epidermis, fat body, and midgut, were dissected from transgenic $6^{\text {th }}$ instar larvae. They were then processed and subjected to luciferase activity assay as described above. Three biological replicates were performed for each treatment.

\section{P450 activity assay in tissues of transgenic insects}

Midgut and fat body were dissected from the wild-type and transgenic FAW $6^{\text {th }}$ instar larvae and homogenized in 1.7-ml tubes containing ice-cold $0.1 \mathrm{M}$ sodium phosphate buffer $(\mathrm{pH} 7.5)$ with $1 \mathrm{mM}$ EDTA, $1 \mathrm{mM}$ PMSF, $0.1 \mathrm{mM}$ DTT, and $1 \mathrm{mM}$ PTU. Then the homogenates were clarified two times by centrifugation at $1,000 \times g$ for $5 \mathrm{~min}$ at $4{ }^{\circ} \mathrm{C}$. Protein concentration in the supernatant was measured using the Bradford method. The supernatant was diluted with sodium phosphate buffer to give a final protein concentration of $1 \mathrm{mg} / \mathrm{ml}$. Luminescent P450 activity assays were performed in all-white 96-well plates using commercial P450-Glo substrate Luciferin-Be (Promega, Madison, WI, USA) as described (Inceoglu et al. 2009). To initiate the P450 activity assay, $50 \mu \mathrm{M}$ of Luciferin-Be substrate was added into 
each well containing $40 \mu \mathrm{L}$ of homogenate supernatant, and $0.1 \mathrm{M}$ sodium phosphate buffer mentioned above was added to each reaction to reach a final volume of $100 \mu \mathrm{L}$. The luminescent reactions were incubated at room temperature for $1 \mathrm{hr}$. Then, $25 \mu \mathrm{L}$ of the reaction mixture was transferred to a new well. After adding $25 \mu \mathrm{L}$ of luciferin detection reagent, the reactions were incubated at $27^{\circ} \mathrm{C}$ for an additional $20 \mathrm{~min}$. The luminescence was quantified using SpectraMax i3x (San Jose, CA, USA). The average relative luminescence unit (RLU) values from wells containing all assay components except the tissue were used as a blank. Three biological replicates were performed for each treatment.

\section{Deltamethrin bioassay}

Bioassays were conducted with newly $1^{\text {st }}$ instar larvae using a standard leaf disk method (Yang et al. 2017). The deltamethrin (EMD Millipore Crop., USA) was diluted using $0.1 \%$ Triton X-100 to produce serial concentrations of solution. Brussels sprout leaves were washed and dried at room temperature were cut into small, $\sim 1 \times 0.5 \mathrm{~cm}$ pieces and dipped in the deltamethrin solution for 10 seconds. These leaf discs were air-dried on food wrap film at room temperature for 20-30 min. Leaf discs dipped in sterile distilled water containing $0.1 \%$ Triton $\mathrm{X}-100$ were used as controls. The treated leaf discs were then transferred to 2.0-ml Eppendorf tubes. Fifteen newly molted $1^{\text {st }}$ instar larvae were gently transferred into each tube. The tubes were sealed with cotton and kept at $27 \pm 2{ }^{\circ} \mathrm{C}, 65 \pm 5 \%$ relative humidity, and photoperiod of $16: 8$ (light: dark). Each treatment has three replicates. Mortality was recorded at $48 \mathrm{~h}$ after exposure to deltamethrin. Larvae were considered dead if they failed to move when prodded with a brush.

\section{Statistical analysis}

$\mathrm{LC}_{50}$ values were generated using GraphPad Prism 8. IBM SPSS Statistics 25 was used in statistical analysis. All data were shown as mean \pm SD (standard deviation). Significant differences between the two groups were analyzed using independent samples $t$ test; $p<0.05$ was considered statistically significant.

\section{Results And Discussion}

\section{Identification of midgut-specific promoters in FAW}

In model insects, including D. melanogaster and Bombyx mori, a number of midgut-specific promoters have been identified and used for tissue-specific expression of transgenes (Fischer et al. 1988; Hu et al. 2015; Jiang et al. 2013; Park and Kwon 2011; Zeng et al. 2010). Midgut-specific promoters were also identified in several mosquito species (Moreira et al. 2000; Nolan et al. 2011; Skavdis et al. 1996; Zhao et al. 2014). Although some midgut-specific promoters showed cross-species activity (Skavdis et al. 1996), it is still not feasible to use midgut-specific promoters in non-host insects. Information on midgut-specific promoters in non-model pest insects is not available.

Several highly active promoters from FAW have been identified, and their activity in vivo was determined (Chen et al. 2020a). In this study, a group of eight highly expressed genes in the midgut and other tissues 
and a group of 16 genes that are expressed only in the midgut were identified in RNA-Seq data. The mRNA levels of these genes were investigated by RT-qPCR. All eight highly expressed genes showed significantly higher expression levels in the midgut than in the other tissues. The expression levels of $S f C P H 38$ in the midgut is 2,048,465-fold higher than in other tissues (Figure 1A). In the group of midgutspecific genes, two genes, SfZCPase2 and SfmALP, did not express in other tissues. However, their expression levels in the midgut are much lower than the other 14 genes. Among these 14 genes, SfTrypsinC showed the highest specificity in the midgut, 1,842,596-fold higher than in other tissues (Figure 1B). The overall relative expression levels of 16 midgut-specific genes are much lower than those of eight highly expressed genes. NCBI accession numbers of these genes are provided in Table S2.

Following the method described in our previous paper (Chen et al. 2020a), the putative promoter regions of 11 midgut specific genes, including SfTrypsinC/P844, SfSP/P415, SfLipase3/P1558, SfCBP-I/P360, SfCalphotin/P799, SfTrypsin/P1705, SfCptlike/P857, SfMG17/P2000, SfSP11/P2000, SfSP38/P2000, and SfCPH38/P2000 were amplified from the genomic DNA and cloned.

\section{Determination of the specificity of candidate FAW midgut promoters in vivo using baculovirus}

Two FAW ovarian cell lines, Sf9 and Sf21, and one FAW midgut cell line Sf17 were used to investigate the FAW promoter activity. The FAW promoters from highly expressed genes displayed weak or no activity in these cell lines (Bleckmann et al. 2015; Chen et al. 2020a). The cell lines are not ideal for investigating the activity of tissue-specific promoters, which is likely due to the lack of essential transcription factors required for the activation of tissue-specific genes. Also, the promoter activity in the cell line may not always match with that in the insect in vivo. Recently, the baculoviruses were used to evaluate the performance of B. mori promoters in vivo (Tian et al. 2018; Zhang et al. 2015; Zhao et al. 2014).

To test whether the baculovirus could be used to measure the promoter activity in FAW, a reporter baculovirus expressing the luciferase gene under the control of the promoter region of an early baculovirus gene, pag1, was constructed. This reporter baculovirus, pag1:Luc, was then added to the Sf9 cells and Sf17 cells. Both cells showed luciferase activity at $24 \mathrm{hr}$ post-infection, and the luciferase activity in virus-infected Sf 9 cells is much higher than in virus-infected Sf17 cells (Figure S1). The virus was then injected into the newly molted 6th instar larvae. Midgut and other tissues were dissected on 1, 2, 3,4 , and 5 days post-injection. In the midgut, the maximum luciferase activity was detected on day 3 . In samples containing all other tissues, the maximum luciferase activity was detected on day 4 (Figure S2). The luciferase activity was not detected in uninfected wild-type larvae or those infected with another reporter baculovirus expressing EGFP (data not shown). These results indicated that reporter baculovirus could be employed to evaluate FAW promoter performance in vivo. Since the baculoviruses could quickly spread to different tissues of FAW, we hypothesized that the reporter baculovirus carrying tissue-specific promoter may support tissue-specific transgene expression in infected FAW larvae. The reporter baculoviruses expressing the luciferase controlled by 11 candidate midgut-specific promoters were 
generated. The plaque-forming units (PFU) of each reporter baculovirus was determined by RT-qPCR (Figure S3). Unlike the pag1:Luc baculovirus, most of these 11 reporter baculoviruses showed higher luciferase activity in the Sf17 cells than in Sf9 cells (Figure 2A). These reporter baculoviruses were injected into the hemocoel of FAW larvae, and the luciferase activity in the midgut and other tissues was measured on day 3 after injection. The result showed that weak or no luciferase activity was detected in the midgut while the other tissues showed some luciferase activity (Figure 2B). The reporter baculoviruses failed to show the expected midgut-specific expression of the reporter gene, which is likely due to the injection of the virus into the hemocoel. Also, baculovirus infection may have altered cellular gene expression patterns (Blissard 1996; Clem and Passarelli 2013), making endogenous promoters inactive and/or changes in their tissue specificity.

\section{Determination of the specificity of candidate FAW midgut promoters in vivo using transgenic insects}

In recent years, the transgenic insects have been employed to evaluate the $B$. mori and $A$. aegypti promoters in vivo (Anderson et al. 2010; Deng et al. 2013; Jiang et al. 2013; Moreira et al. 2000; Totten et al. 2013; Xu et al. 2019; Xu et al. 2014). However, germline transformation technologies are not well established in non-model insects, likely due to the low transformation efficiency and difficulty of delivering transformation components into the fresh embryos. We recently established piggyBac-based transformation system in FAW (Chen et al. 2020b), which was used to test FAW promoters in vivo.

A piggyBac vector, expressing a marker protein EGFP under the control of ie1 promoter, was used to make the reporter vectors. The promoters from the genes that are highly expressed in the midgut were cloned into this piggyBac vector to drive the luciferase expression. The hr5 enhancer, which is capable of boosting promoter activity, was placed between ie1 promoter and chosen midgut-specific promoter to enhance the expression of both the EGFP and the luciferase (Figure 3A). Four reporter vectors, hr5SfSP38/P2000:Luc, hr5-SfMG17/P2000:Luc, hr5-SfCalphotin/P799:Luc, and hr5-SfCPH38/P2000:Luc were produced. A mixture of each reporter vector and the mRNA of hyperactive transposase, which was reported to increase the transformation efficiency in several insect species (Eckermann et al. 2018; Otte et al. 2018), was injected into 2000 fresh embryos collected within $6 \mathrm{hr}$ after oviposition. Forty-six transgenic hr5-SfSP38/P2000: Luc neonate larvae, 31 transgenic hr5-SfMG17/P2000:Luc neonate larvae, 56 transgenic hr5-SfCalphotin/P799: Luc neonate larvae and 41 transgenic hr5-SfCPH38/P2000:Luc neonate larvae were identified based on the EGFP marker gene expression in the G1 generation (Table 1). The adults developed from $\mathrm{G} 1$ positive larvae were crossed with wild-type adults to produce $\mathrm{G} 2$ generation. The positive transgenic larvae from all four lines showed strong GFP signals in G2 (Figure $3 \mathrm{~B}$ ), indicating that the transgenic insertions are inheritable. We then tested the luciferase activity in four tissues, including the head, epidermis, fat body, and midgut dissected from each transgenic line. The results showed that the midgut has significantly higher luciferase activity than in other tissues. The luciferase activity in the midgut is 1,731-, 1,558-, 2,337-, and 14,124-fold higher than in fat body of hr5SfSP38/P2000:Luc, hr5-SfMG17/P2000:Luc, hr5-SfCalphotin/P799:Luc, and hr5-SfCPH38/P2000:Luc 
transgenic larvae, respectively (Figure 3C). SfCalphotin/P799 promoter displayed higher activity in the midgut and fat body than the other three promoters tested. SfCPH38/P2000 promoter showed the best specificity in the midgut. Low luciferase activity was also detected in other tissues, indicating the weak activity of these promoters in other tissues (Figure 3C).

Table 1

Germline transformation of transgenic constructs.

\begin{tabular}{|lllll|}
\hline Plasmids & $\begin{array}{l}\text { Injected eggs } \\
\text { (n) }\end{array}$ & $\begin{array}{l}\text { G0 Larvae } \\
\text { (n) }\end{array}$ & $\begin{array}{l}\text { G0 Pupae } \\
\text { (n) }\end{array}$ & $\begin{array}{l}\text { G1 Positive } \\
\text { (n) }\end{array}$ \\
\hline hr5-SfSP38/P2000: Luc & $\sim 2200$ & $\sim 800$ & $\sim 600$ & 46 \\
\hline hr5-SfMG17/P2000: Luc & $\sim 2400$ & $\sim 900$ & $\sim 700$ & 31 \\
\hline hr5-SfCalphotin/P799: Luc & $\sim 2400$ & $\sim 1000$ & $\sim 700$ & 56 \\
\hline hr5-SfCPH38/P2000: Luc & $\sim 2000$ & $\sim 800$ & $\sim 600$ & 41 \\
\hline SfCPH38/P2000: Luc & $\sim 2000$ & $\sim 700$ & $\sim 600$ & 29 \\
\hline hr5-SfCPH38/P2000: CYP321A8 & $\sim 2400$ & $\sim 600$ & $\sim 500$ & 38 \\
\hline SfCPH38/P2000: CYP321A8 & $\sim 2400$ & $\sim 800$ & $\sim 600$ & 79 \\
\hline
\end{tabular}

As promoter fused with hr5 enhancer showed elevated activity (Chen et al. 2020a), we hypothesized that removing the hr5 enhancer might decrease the promoter activity in other tissues and increase its specificity in the midgut. To test this hypothesis, a new reporter vector, SfCPH38/P2000:Luc, was constructed (Figure 3A) injected into 2000 eggs. Twenty-nine EGFP-positive neonate larvae were obtained (Table 1). The EGFP signals in transgenic SfCPH38/P2000:Luc G2 positive larvae were not bright as transgenic hr5-SfCPH38/P2000:Luc G2 positive larvae (Figure 3B), as hr5 enhancer also boosts ie1 promoter activity resulting in the production of more EGFP protein. The luciferase activity in the midgut is 13,152-fold higher than in the fat body of SfCPH38/P2000:Luc larvae. Also, the luciferase activity in SfCPH38/P2000:Luc larval tissues is lower than in the tissues of hr5-SfCPH38/P2000 Luc larvae (Figure $3 \mathrm{C})$. These results suggest that hr5 enhancer could significantly enhance the activity, but not the midgutspecificity, of $\mathrm{CPH} 38$ promoter.

\section{Midgut-specific promoter mediated over-expression of P450 confers deltamethrin tolerance to S. frugiperda}

P450 enzymes metabolize insecticides and plant toxins (Dermauw et al. 2020; Feyereisen 2012), and their overexpression is involved in insecticide resistance (Feyereisen 2012; Jiang et al. 2015). Although many P450 genes were found in the genome of FAW (Gui et al. 2020), little is known about their function in insecticide resistance. A recent study revealed that overexpression of CYP321A8 in Spodoptera exigua, a close relative of $S$. frugiperda, conferred resistance to deltamethrin (Hu et al. 2021). The insect midgut is the major organ encountering plant toxins and insecticides (Hakim et al. 2010; Smagghe and Tirry 
2001). Transgenic overexpression of P450 genes in the midgut likely confers tolerance to insecticides. To test whether the identified midgut-specific promoters could be used in transgenic FAW to study insecticide resistance, SfCPH38/P2000 promoter was used to drive expression of SfCYP321A8 gene in the midgut.

The SfCYP321A8 gene was cloned. Two vectors, SfCPH38/P2000:CYP321A8 and hr5SfCPH38/P2000:CYP321A8 were produced and injected into embryos (Figure 4A). Seventy-nine transgenic SfCPH38/P2000:CYP321A8 neonate larvae and 38 transgenic hr5-

SfCPH38/P2000:CYP321A8 neonate larvae were identified based on the EGFP marker gene expression in the G1 generation (Table 1). The EGFP signals in transgenic SfCPH38/P2000:CYP321A8 G2 positive larvae are less bright than the transgenic hr5-SfCPH38/P2000:CYP321A8 G2 positive larvae (Figure 4B).

The relative mRNA levels of SfCYP321A8 were determined in the head, midgut, fat body, and epidermis dissected from the 6th instar larvae of wild-type and two G2 SfCYP321A8 transgenic insects. The highest expression levels of SFCYP321A8 mRNA were detected in the midgut of both transgenic lines. An increase by 155.5- and 287.6-fold in the SfCPH38/P2000:CYP321A8 and hr5-SfCPH38/P2000:CYP321A8 mRNA levels transgenic larvae, respectively, when compared to their levels in the wild-type larvae were detected (Figure 4C). The expression also increased by 6.3- and 10.6-fold in the fat body of SfCPH38/P2000:CYP321A8 and hr5-SfCPH38/P2000:CYP321A8 transgenic larvae, respectively. In the epidermis of transgenic hr5-SfCPH38/P2000:CYP321A8 larvae, the mRNA level of SfCYP321A8 was slightly higher than in wild-type and control transgenic larvae. No significant changes in SfCYP321A8 expression were detected in the epidermis of both transgenic larvae (Figure 4C). To test whether overexpressing of SfCYP321A8 in the midgut leads to an overall increase in total P450 activity in the midgut of the transgenic animals; the midgut and fat body were tested for P450 activity. The total P450 activity in the midgut dissected from SfCPH38/P2000:CYP321A8 and hr5-SfCPH38/P2000: CYP321A8 transgenic larvae increased by 1.41 - and 1.48 -fold, respectively, when compared to the P450 activity in the wild-type larvae. However, no increase in P450 activity was detected in the fat body of both transgenic larvae (Figure 4D). These results suggest that SfCPH38/P2000 promoter is capable of driving midgutspecific expression of SfCYP321A8.

The expression levels of both SfCPH38 and SfCYP321A8 were investigated in the eggs and 1st instar larvae of wild-type and transgenic lines. Expression of SFCYP321A8 in 1st instar larvae of both transgenic lines gradually increased similar to the $\mathrm{SfCPH} 38$ gene expression (Figure S4). Leaf-disc assays were performed to evaluate the efficacy of deltamethrin in wild-type and transgenic 1 st instar larvae. The results showed that the $\mathrm{LC}_{50}$ of deltamethrin in SfCPH38/P2000:CYP321A8 and hr5-SfCPH38/P2000: CYP321A8 larvae increased by 4.2- and 4.5-fold, respectively, when compared to the wild-type larvae (Figure 4E). These data suggest that transgenic overexpression of SfCYP321A8 in the midgut increased deltamethrin tolerance in FAW larvae.

Identification and characterization of midgut-specific promoters from a non-model insect, FAW are included in this paper. The de novo mining of tissue-specific promoters needs gene expression data, which is not available for many insects. Since the information on many tissue-specific promoters from 
the model insects is available, it may be possible to identify the homologous promoters in the target nonmodel insects. In the lepidopteran model insect, B. mori, promoter of APN gene, is only active in the midgut (Jiang et al. 2015). We found six APN genes in S. frugiperda. RT-qPCR analysis revealed midgutspecific expression of all APN genes (Figure S5). The candidate promoter region of the SfAPN6 gene was cloned and used to drive the expression of SfCYP321A8. Transgenic SfAPN6/P1949:CYP321A8 animals were obtained (Table S3, Figure S6A). However, the mRNA levels of SfCYP321A8 only increased by 2.3fold in the midgut of this transgenic line than in the wild-type (Figure S6B). Also, this transgenic line showed similar levels of deltamethrin susceptibility as the wild-type (Figure S6C). The candidate promoter region of $S F S P 11$, homolog of a midgut-specific serine protease in B. mori (Liu et al. 2017), was also tested to drive expression of SfCYP321A8 in animals (Table S3, Figure S6A). A 16.7-fold increase of SfCYP321A8 mRNA levels was detected in the midgut of transgenic animals than in the wild-type (Figure S6B). However, no increase in deltamethrin tolerance was observed (Figure S6C). Since these two midgutspecific promoters of FAW were identified from information from $B$. mori did not show good activity as $\mathrm{SfCPH} 38 / \mathrm{P} 2000$ promoter, our hypothesis on using information from model insects to identify promoters in non-model insects is not supported. We used $\sim 2 \mathrm{kp}$ region upstream to the ATG of each gene as candidate promoter region. The low activity of SfAPN6 and SfSP11 promoters might be because this region did not include complete promoter.

Transgenic reporter insects have been used to investigate the promoter performance in vivo. However, germline transformation technologies are not available in most non-model insects. We tried to use baculoviruses to deliver the reporter gene expression cassettes to different tissues of FAW larvae but failed to determine the activity of midgut-specific promoters in vivo. A recent report revealed one baculovirus species, Autographa californica multiple nucleopolyhedrovirus (AcMNPV), is an efficient vector for gene delivery into several mosquito species; it can transduce both larvae and adults with little or no tissue barriers and without obvious negative effects (Naik et al. 2018). It seems that baculovirus could be used to investigate the promoter performance in non-permissive hosts in vivo.

In conclusion, transgenic reporter insects were successfully used to determine the in vivo performance of midgut-specific promoters in an important agricultural pest, FAW. Midgut-specific promoter was used to investigate the role of P450 in insecticide resistance. This work could serve as a model for exploring other tissue-specific promoters, which will benefit functional genomic studies in FAW and other non-model insects.

\section{Declarations}

\section{Acknowledgments}

We thank Jeff Howell from University of Kentucky for help with FAW rearing, and Dr. Anjiang Tan of Shanghai institute of plant physiology and ecology, China for the gift of piggyBac vector,and Dr. Cynthia L. Goodman from USDA, ARS, BCIRL at Columbia, MO, for the gift of SfMG_0617 cells. This material is based on work supported by the National Science Foundation I/UCRC, the Center for Arthropod 
Management Technologies, under Grant No. IIP1821936, and by industry partners, and the National Institute of Food and Agriculture, US Department of Agriculture (under HATCH Project 2353057000).

All authors certify that they have no affiliations with or involvement in any organization or entity with any financial interest or non-financial interest in the subject matter or materials discussed in this manuscript.

All data generated or analysed during this study are included in this published article [and its supplementary information files].

\section{References}

1. Anderson MA, Gross TL, Myles KM, Adelman ZN (2010) Validation of novel promoter sequences derived from two endogenous ubiquitin genes in transgenic Aedes aegypti. Insect molecular biology 19:441-449

2. Bleckmann M et al (2015) Genomic analysis and isolation of RNA polymerase II dependent promoters from spodoptera. frugiperda PloS One 10:e0132898

3. Blissard GW (1996) Baculovirus-insect cell interactions Cytotechnology 20:73-93

4. Carvalho RA, Omoto C, Field LM, Williamson MS, Bass C (2013) Investigating the molecular mechanisms of organophosphate and pyrethroid resistance in the fall armyworm Spodoptera. frugiperda PLoS One 8:e62268

5. Chen X, Chereddy SC, Gurusamy D, Palli SR (2020a) Identification and characterization of highly active promoters from the fall armyworm. Spodoptera frugiperda Insect Biochemistry Molecular Biology 126:103455

6. Chen X, Koo J, Gurusamy D, Mogilicherla K, Reddy Palli S (2020b) Caenorhabditis elegans systemic RNA interference defective protein 1 enhances RNAi efficiency in a lepidopteran insect, the fall armyworm, in a tissue-specific manner RNA biology:1-9

7. Clem RJ, Passarelli AL (2013) Baculoviruses: sophisticated pathogens of insects. PLoS Pathog 9:e1003729

8. Deng D, Xu H, Wang F, Duan X, Ma S, Xiang Z, Xia Q (2013) The promoter of Bmlp3 gene can direct fat body-specific expression in the transgenic silkworm. Bombyx mori Transgenic research 22:10551063

9. Dermauw W, Van Leeuwen T, Feyereisen R (2020) Diversity and evolution of the P450 family in arthropods Insect biochemistry and molecular biology 127:103490

10. Diez-Rodriguez G, Omoto C (2001) Inheritance of lambda-cyhalothrin resistance in Spodoptera frugiperda (JE Smith)(Lepidoptera: Noctuidae). Neotropical Entomology 30:311-316

11. Eckermann $\mathrm{KN}$ et al (2018) Hyperactive piggyBac transposase improves transformation efficiency in diverse insect species. Insect Biochem Mol Biol 98:16-24

12. Engel P, Moran NA (2013) The gut microbiota of insects-diversity in structure and function FEMS microbiology reviews 37:699-735 
13. Feyereisen R (2012) Insect CYP genes and P450 enzymes. In: Insect molecular biology and biochemistry. Elsevier, pp 236-316

14. Fischer JA, Giniger E, Maniatis T, Ptashne M (1988) GAL4 activates transcription in. Drosophila Nature 332:853-856

15. Gui $\mathrm{F}$ et al (2020) Genomic and transcriptomic analysis unveils population evolution and development of pesticide resistance in fall armyworm Spodoptera frugiperda Protein \& Cell:1-19

16. Hakim RS, Baldwin K, Smagghe G (2010) Regulation of midgut growth, development, and metamorphosis. Ann Rev Entomol 55:593-608

17. Harrop TW, Pearce SL, Daborn PJ, Batterham P (2014) Whole-genome expression analysis in the third instar larval midgut of Drosophila melanogaster G3: Genes, Genomes. Genetics 4:2197-2205

18. Hu B et al (2021) Changes in both trans-and cis-regulatory elements mediate insecticide resistance in a lepidopteron pest. Spodoptera exigua PLoS genetics 17:e1009403

19. Hu C, Wang F, Ma S, Li X, Song L, Hua X, Xia Q (2015) Suppression of intestinal immunity through silencing of TCTP by RNAi in transgenic silkworm. Bombyx mori Gene 574:82-87

20. Hung R-J et al (2020) A cell atlas of the adult Drosophila midgut Proceedings of the National Academy of Sciences 117:1514-1523

21. Inceoglu A, Waite T, Christiansen J, McAbee R, Kamita S, Hammock B, Cornel A (2009) A rapid luminescent assay for measuring cytochrome $\mathrm{P} 450$ activity in individual larval Culex pipiens complex mosquitoes (Diptera: Culicidae. J Med Entomol 46:83-92

22. Jiang $L$ et al (2013) Identification of a midgut-specific promoter in the silkworm Bombyx mori Biochemical and biophysical research communications 433:542-546

23. Jiang $L$ et al (2015) The 5'-UTR intron of the midgut-specific BmAPN4 gene affects the level and location of expression in transgenic silkworms Insect biochemistry and molecular biology 63:1-6

24. Liu HW et al (2017) A midgut-specific serine protease, BmSP36, is involved in dietary protein digestion in the silkworm. Bombyx mori Insect science 24:753-767

25. Lucchetta EM, Ohlstein B (2012) The Drosophila midgut: a model for stem cell driven tissue regeneration Wiley Interdisciplinary Reviews. Dev Biol 1:781-788

26. Montezano DG et al (2018) Host plants of Spodoptera frugiperda (Lepidoptera: Noctuidae) in the Americas African Entomology 26:286-300

27. Moreira LA, Edwards MJ, Adhami F, Jasinskiene N, James AA, Jacobs-Lorena M (2000) Robust gutspecific gene expression in transgenic Aedes aegypti mosquitoes Proceedings of the National Academy of Sciences 97:10895-10898

28. Naik NG, Lo Y-W, Wu T-Y, Lin C-C, Kuo S-C, Chao Y-C (2018) Baculovirus as an efficient vector for gene delivery into mosquitoes. Scientific reports 8:1-14

29. Nolan T, Petris E, Müller H-M, Cronin A, Catteruccia F, Crisanti A (2011) Analysis of two novel midgutspecific promoters driving transgene expression in Anopheles stephensi mosquitoes PloS one $6: \mathrm{e} 16471$ 
30. Omoto C et al (2016) Field-evolved resistance to Cry1Ab maize by Spodoptera frugiperda in Brazil. Pest Manag Sci 72:1727-1736

31. Otte M, Netschitailo O, Kaftanoglu O, Wang Y, Page R Jr, Beye M (2018) Improving genetic transformation rates in honeybees Scientific reports 8:1-6

32. Park J-H, Kwon JY (2011) Heterogeneous expression of Drosophila gustatory receptors in enteroendocrine cells. PLoS One 6:e29022

33. Parthasarathy R, Palli SR (2007) Developmental and hormonal regulation of midgut remodeling in a lepidopteran insect. Heliothis virescens Mechanisms of development 124:23-34

34. Skavdis G, Sidén-Kiamos I, Müller H, Crisanti A, Louis C (1996) Conserved function of anopheles gambiae midgut-specific promoters in the fruitfly The. EMBO journal 15:344-350

35. Smagghe G, Tirry L (2001) Insect midgut as a site for insecticide detoxification and resistance. In: Biochemical sites of insecticide action and resistance. Springer, pp 293-321

36. Tian S, Zhang Z, Zhang R, Liu Q, Xu S, Gong J, Hou Y (2018) Analysis of cysteine protease inhibitor gene (BmCPI) promoter activity in silkworms using bac-to-bac baculovirus systems Bioscience, biotechnology, and biochemistry 82:1488-1496

37. Totten DC, Vuong M, Litvinova OV, Jinwal UK, Gulia-Nuss M, Harrell RA, Beneš H (2013) Targeting gene expression to the female larval fat body of transgenic Aedes aegypti mosquitoes Insect molecular biology 22:18-30

38. Wu Y, Parthasarathy R, Bai H, Palli SR (2006) Mechanisms of midgut remodeling: juvenile hormone analog methoprene blocks midgut metamorphosis by modulating ecdysone action. Mechanisms of development 123:530-547

39. Xu J et al (2019) Identification of a germline-expression promoter for genome editing in Bombyx mori. Insect Sci 26:991-999

40. Xu J et al (2014) Functional characterization of the vitellogenin promoter in the silkworm. B ombyx mori Insect molecular biology 23:550-557

41. Yang F, Kerns DL, Head GP, Price P, Huang F (2017) Cross-resistance to purified Bt proteins, Bt corn and Bt cotton in a Cry2Ab2-corn resistant strain of Spodoptera frugiperda. Pest management science 73:2495-2503

42. Zeng X, Chauhan C, Hou SX (2010) Characterization of midgut stem cell-and enteroblast-specific Gal4 lines in. Drosophila Genesis 48:607-611

43. Zhang K et al (2015) Identification and characterization of three novel hemocyte-specific promoters in silkworm Bombyx mori Biochemical and biophysical research communications 461:102-108

44. Zhao G et al (2014) Activation of BmGSTd1 promoter and regulation by transcription factor Krüppel $(\mathrm{Kr})$ in silkworm. Bombyx mori Gene 551:152-159

45. Zhou K, Goodman CL, Ringbauer J, Song Q, Beerntsen B, Stanley D Cellular (2020) Establishment of two midgut cell lines from the fall armyworm, Spodoptera frugiperda (Lepidoptera: Noctuidae) In Vitro. \& Developmental Biology-Animal 56:10-14 
Figures

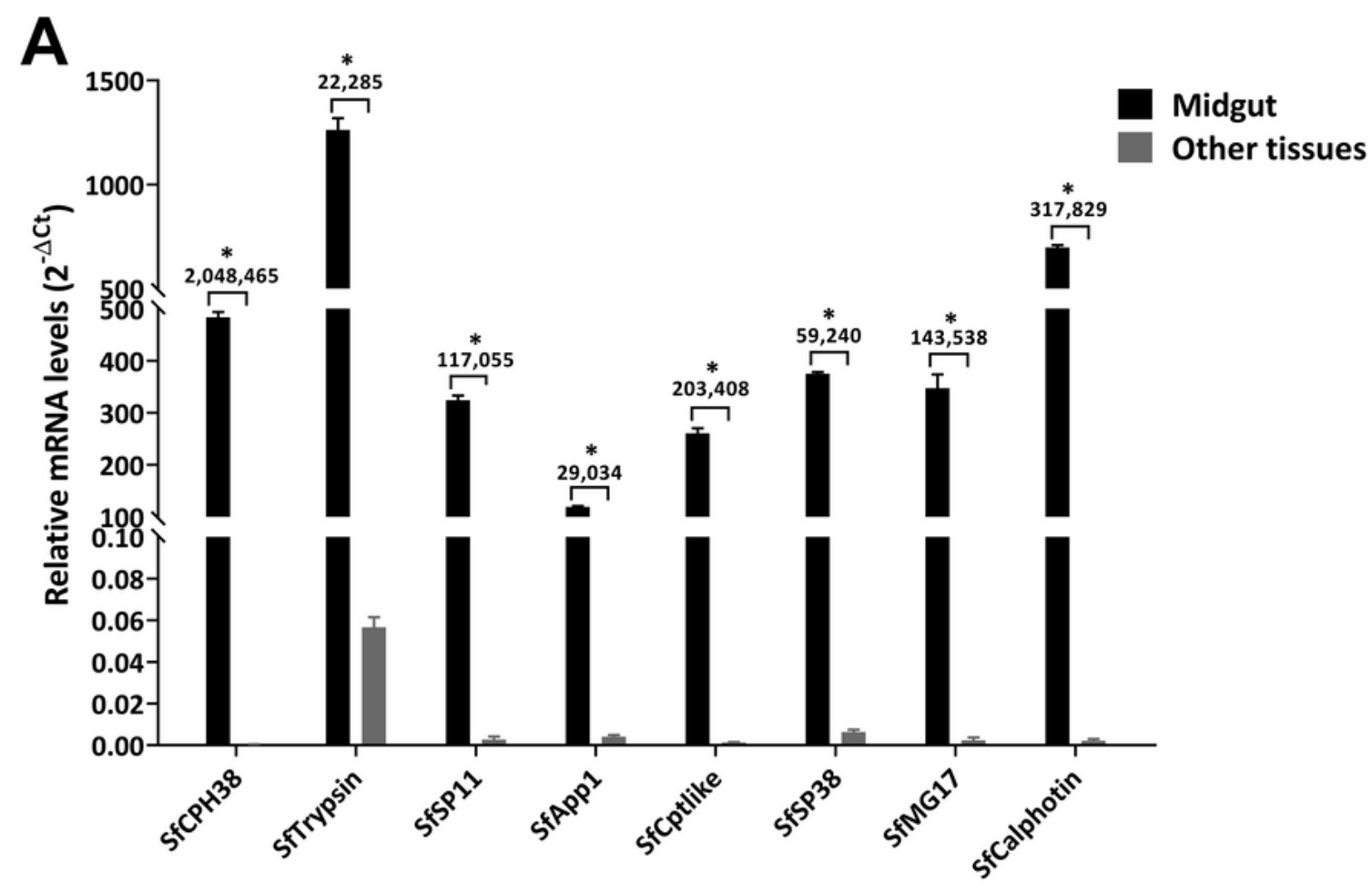

B

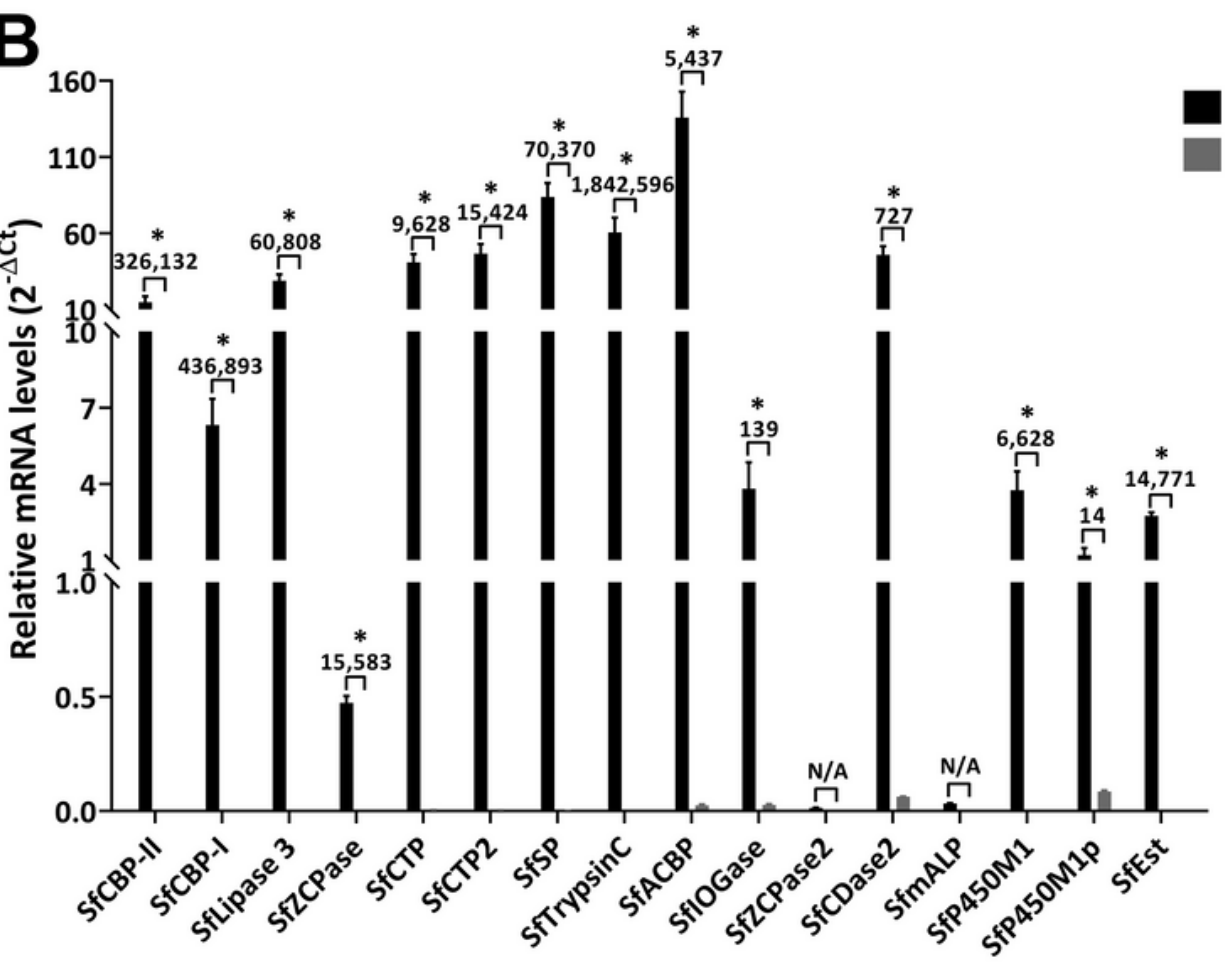

Figure 1

Relative mRNA levels of genes that are highly expressed in the midgut. Midgut-specific genes expressed in the midgut (A) and other tissues (B) of 6th instar FAW larvae. Total RNA was isolated from the midgut and all other tissues, converted to cDNA and the CDNA and gene-specific primers were used in RT-qPCR to 
determine relative mRNA levels using 28S rRNA as the reference gene. Means \pm SD $(n=3)$ are shown. Data were analyzed using student's t-test. *, p<0.05.

A

SfTrypsinC/P844: Luc-

SfSP/P415: Luc-

Sf17

SfLipase3/P1558: Luc-

SfCBP-I/P360: Luc-

SfCalphotin/P799: Luc-

SfTrypsin/P1705: Luc-

SfCptlike/P857: Luc-

SfMG17/P2000: Luc-

SfSP11/P2000: Luc -

SfSP38/P2000: Luc-

SfCPH38/P2000: Luc

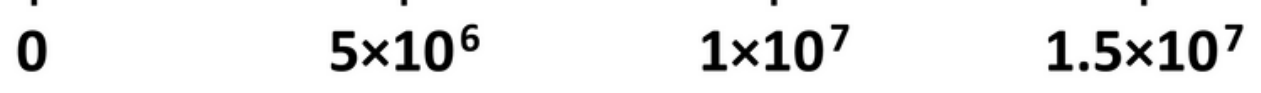

Luciferase activity (RLU/mg Protein)

B

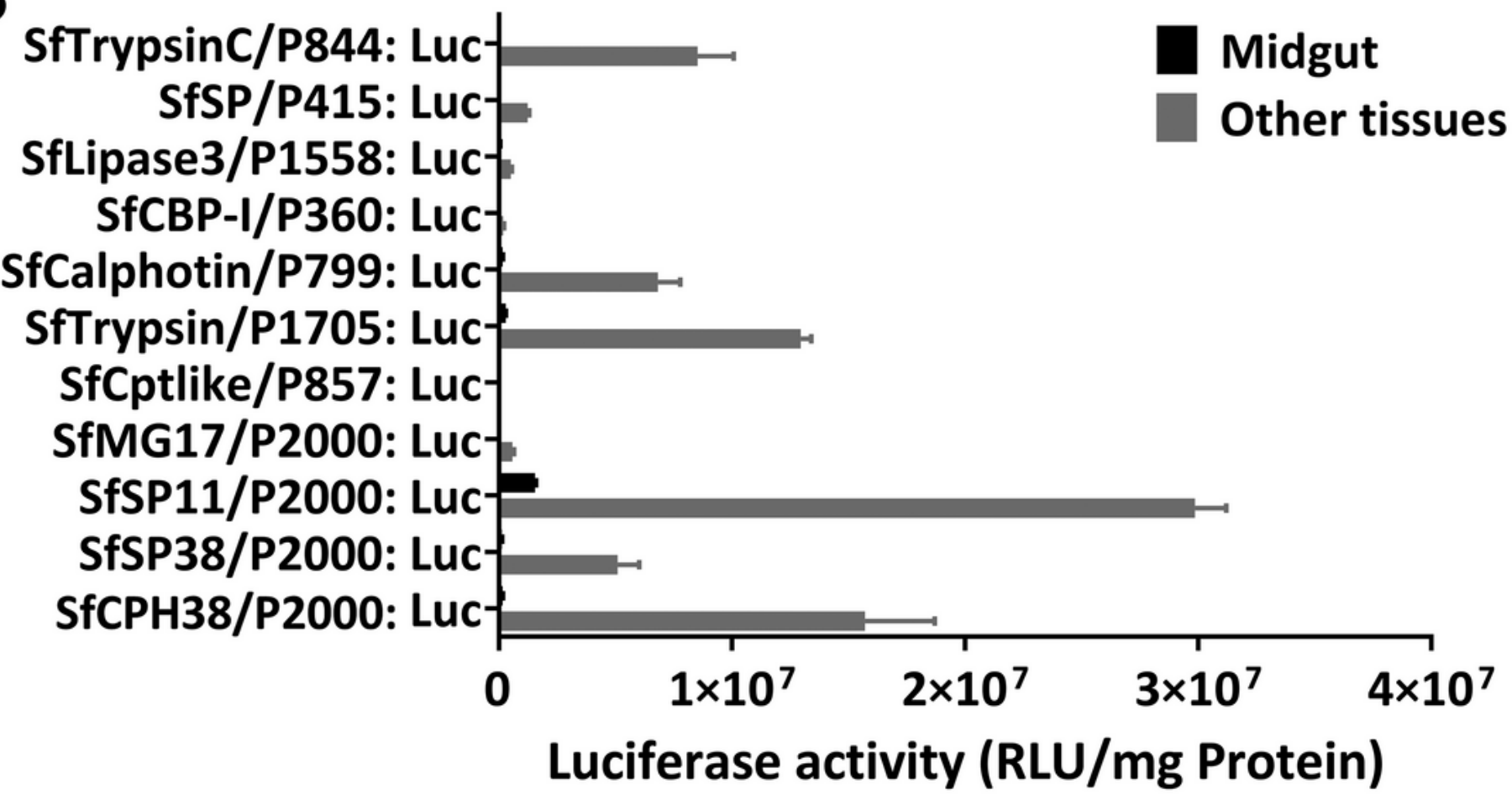

Figure 2

Luciferase activity of reporter baculoviruses in FAW cell lines (A) and tissues (B). Baculoviruses harboring luciferase expression cassette under the control of candidate midgut-specific promoters were generated. The viruses were added to Sf9 cells and Sf17 cell medium. Infected cells were collected for the luciferase 
activity was measured at $24 \mathrm{hr}$ after adding the virus. The newly molted 6 th instar larvae were injected with each virus. Midgut and other tissues dissected on the 3rd-day post-injection were subjected to the luciferase activity assay. Means \pm SD ( $n=4$ for cells, $n=3$ for tissues) are shown.

A

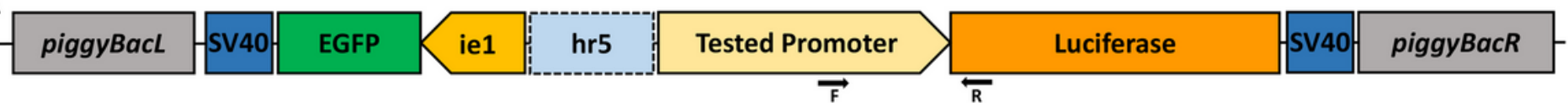

B
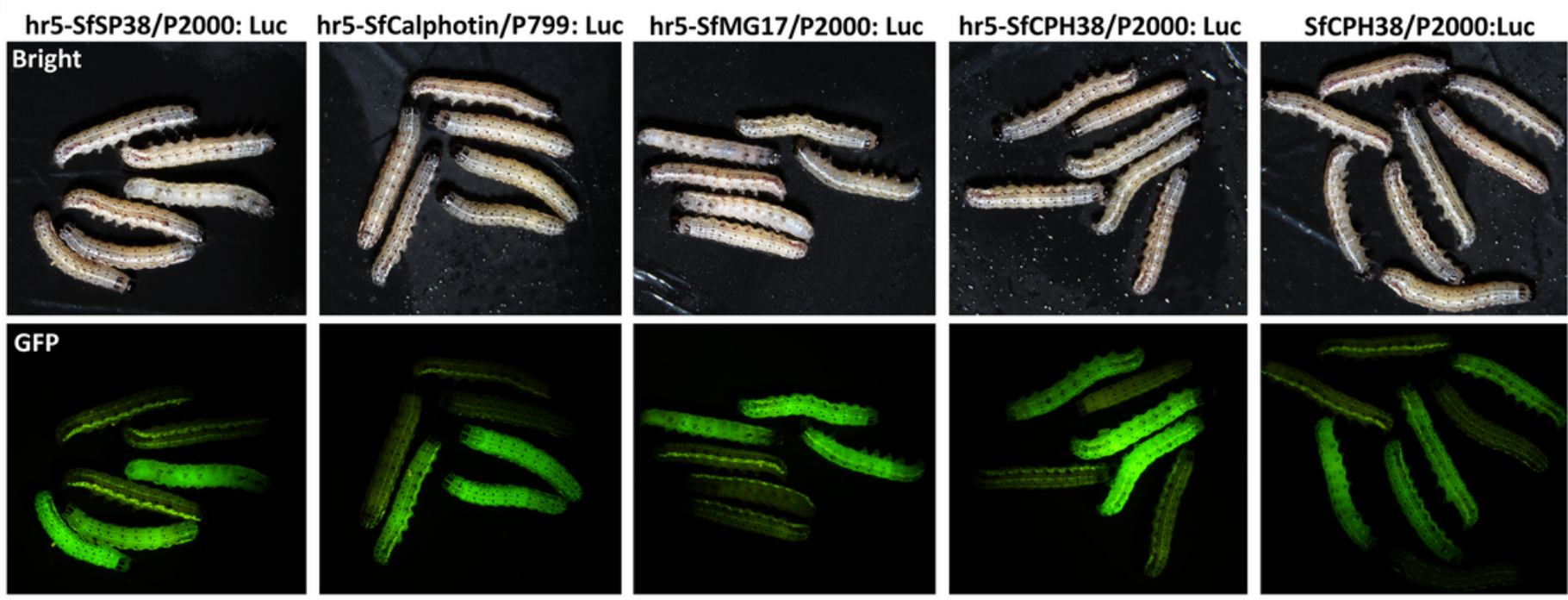

C

hr5-SfSP38/P2000: Luc

hr5-SfCalphotin/P799: Luc
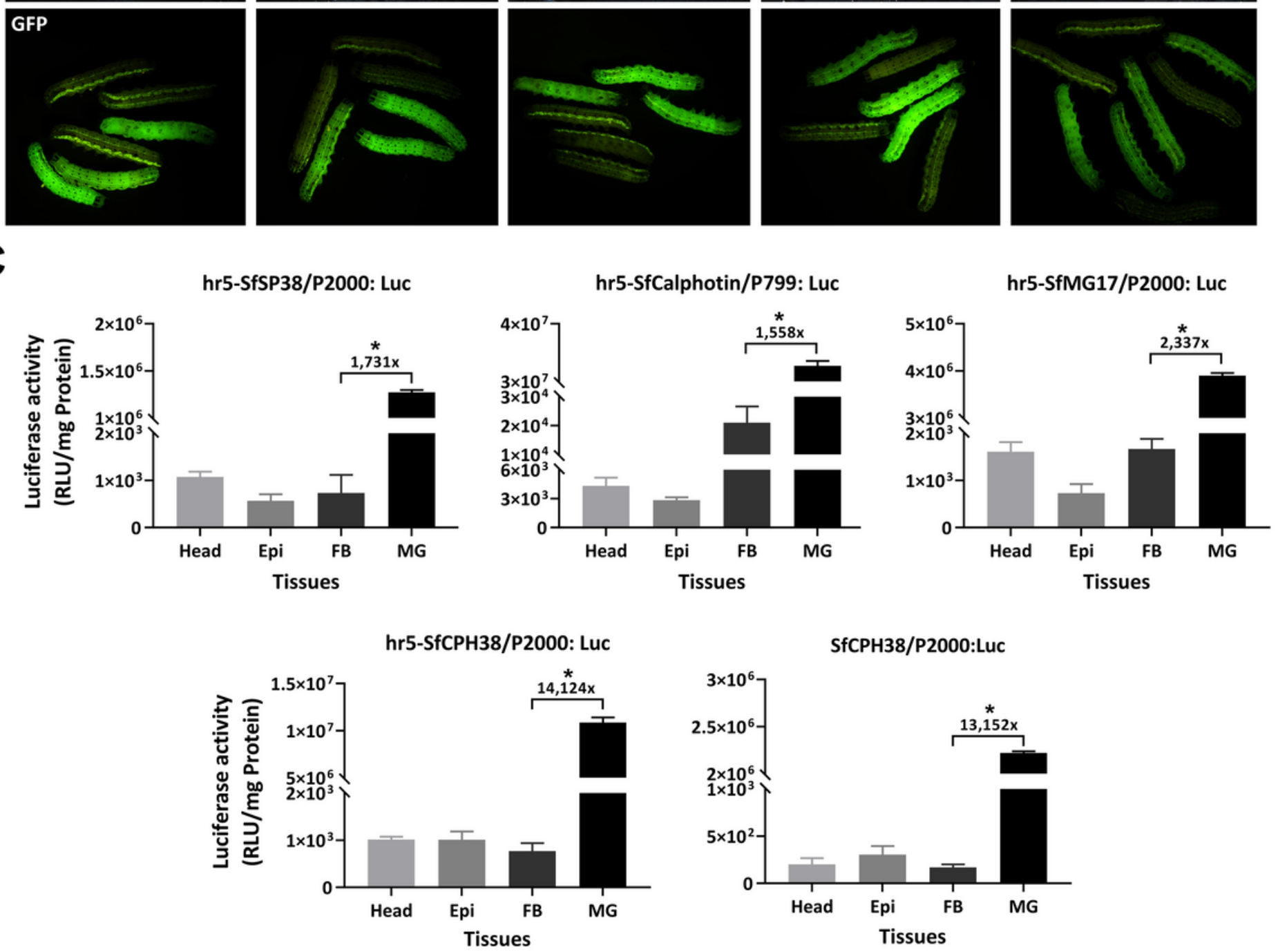

Figure 3

Characterization of transgenic reporter FAW lines to determine midgut-specific promoter activity in vivo. (A) Schematic representation of piggyBac-based reporter constructs. Expression of the luciferase driven by four candidate midgut-specific promoters, SfSP38/P2000, SfCalphotin/P799, SfMG17/P2000, and 
$\mathrm{SfCPH} 38 / \mathrm{P} 2000$. Expression of a marker gene, EGFP, is under the control of ie1 promoter. The hr5 enhancer, highlighted in the dashed quadrangle, inserted between ie1 and midgut-specific promoter in some reporter constructs. (B) Fluorescence in GFP-negative and - positive animals. (C) Luciferase activity in the head, epidermis (Epi), fat body (FB), and midgut (MG) of transgenic reporter animals. Tissues were dissected from transgenic 6 th instar larvae and subjected to luciferase activity assay. Means $\pm S D(n=3)$ are shown. Data were analyzed using student's t-test. *, $p<0.05$.

A

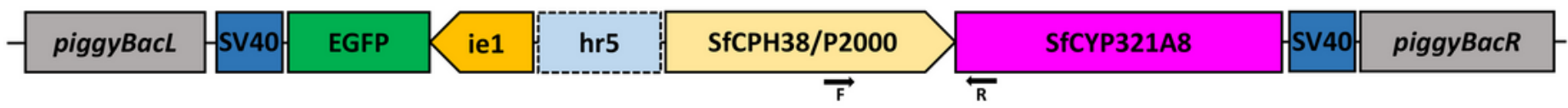

B

SfCPH38/P2000: CYP321A8
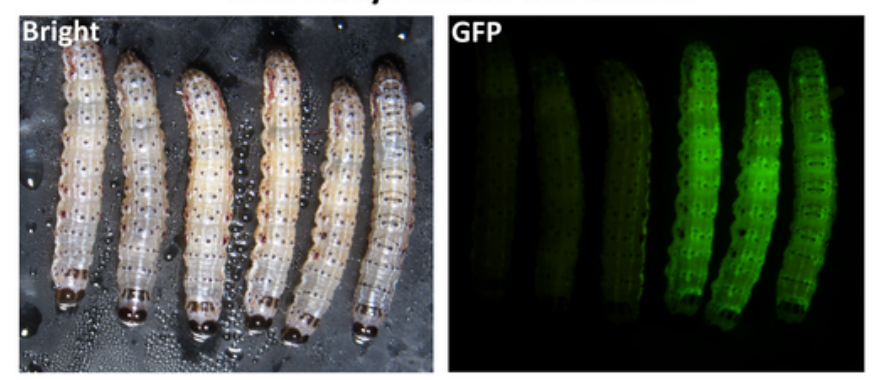

C

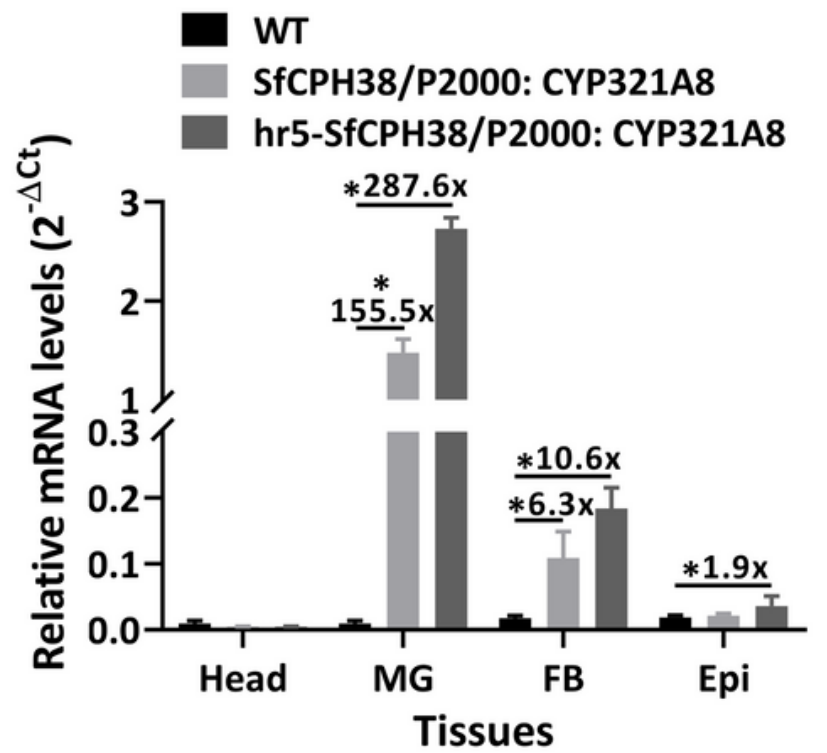

D

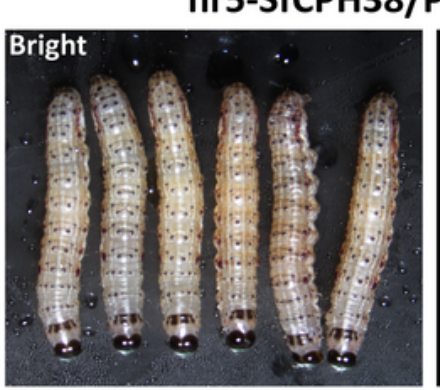

WT
hr5-SfCPH38/P2000: CYP321A8

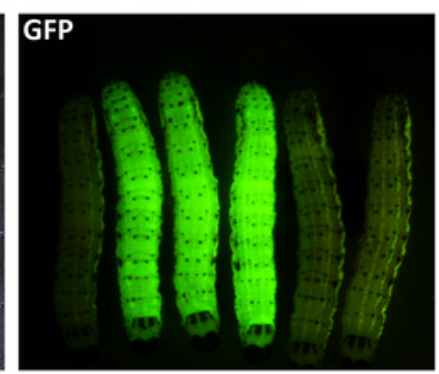

SfCPH38/P2000: CYP321A8

hr5-SfCPH38/P2000: CYP321A8

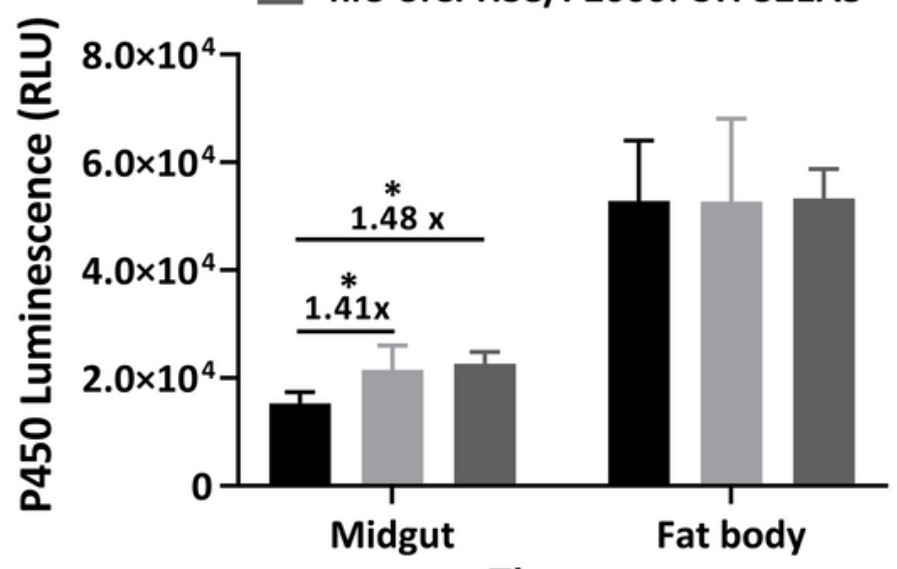

Tissues

E

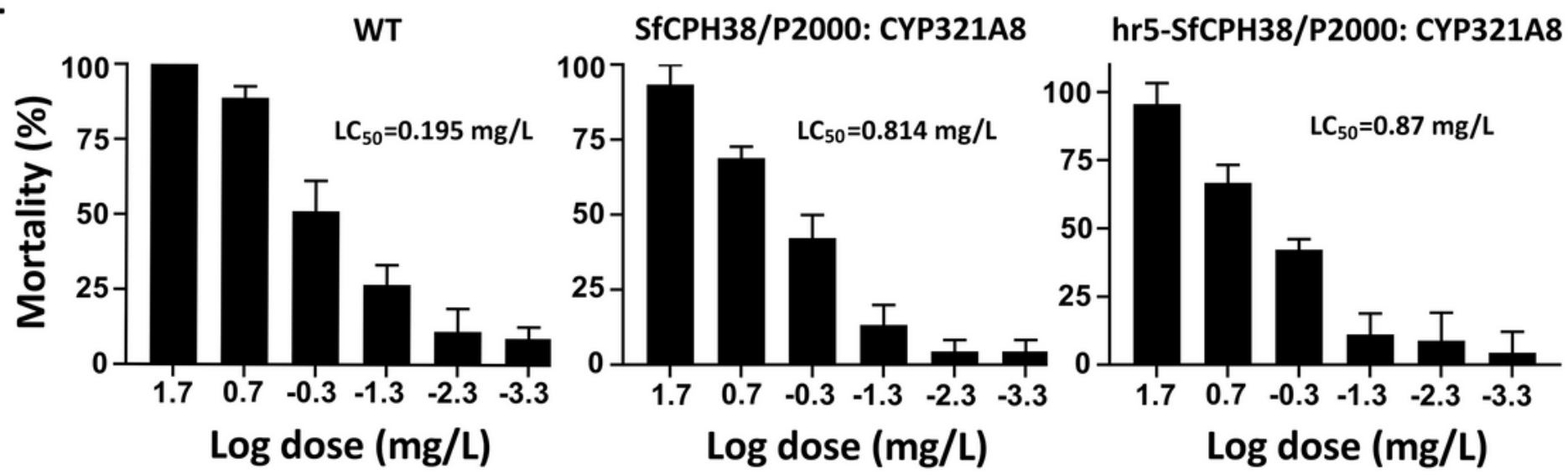

Figure 4 
Characterization of transgenic FAW overexpressing P450 in the midgut. (A) Schematic representation of piggyBac-based constructs expressing SfCYP321A8 under the control of a midgut-specific promoter, SfCPH38/P2000. Expression of a marker gene, EGFP, is under the control of ie1 promoter. The hr5 enhancer, highlighted in the dashed-line rectangle, is located between ie1 and SfCPH38/P2000 promoters. (B) Fluorescence in GFP-negative and -positive animals. (C) Relative mRNA levels of SfCYP321A8 in wild-type (WT) and two transgenic lines. Total RNA was isolated from four tissues, including head, midgut (MG), fat body (FB), and epidermis (Epi). The RNA was converted to CDNA and the CDNA and gene-specific primers were used in RT-qPCR to determine relative mRNA levels using 28S rRNA as the reference gene. Means $\pm S D(n=3)$ are shown. Data were analyzed using student's t-test. *, $p<0.05$. (D) P450-Glo assay of proteins extracted from the midgut and fat body from transgenic animals. The midgut and fat body were dissected from the wild-type and transgenic FAW 6th instar larvae, and homogenates were prepared and assayed using Luciferin-Be as substrate. Mean $\pm S D(n=3)$ are shown. Data were analyzed using student's t-test. *, $p<0.05$. (E) Bioassay of deltamethrin in wild-type and transgenic neonate larvae. The GFP-positive neonates were collected under the fluorescent microscope and fed on leaf discs treated with different concentrations of deltamethrin. Mortality was recorded at 48 hr post-treatment. Mean \pm SD $(n=3)$ are shown.

\section{Supplementary Files}

This is a list of supplementary files associated with this preprint. Click to download.

- Graphicabstract.tif

- Supplementaryinformation.pdf 